\title{
Protocols for entanglement transformations of bipartite pure states
}

\author{
G. Mauro D'Ariano ${ }^{1,2}$ and Massimiliano F. Sacchi ${ }^{1}$ \\ 1 Unità INFM, and Dipartimento di Fisica "A. Volta", via A. Bassi 6, I-27100 Pavia, Ital * and \\ 2 Department of Electrical and Computer Engineering, Northwestern University, Evanston, IL 60208
}

(Dated: October 29, 2018)

\begin{abstract}
We present a general theoretical framework for both deterministic and probabilistic entanglement transformations of bipartite pure states achieved via local operations and classical communication. This framework unifies and greatly simplifies previous works. A necessary condition for "pure contraction" transformations is given. Finally, constructive protocols to achieve both probabilistic and deterministic entanglement transformations are presented.
\end{abstract}

\section{INTRODUCTION}

The transformation of entangled states by means of local operations and classical communication (LOCC) is a key issue in quantum information processing 4], for quantum computation [5], quantum teleportation [6], and quantum cryptography 7]. However, the detrimental effect of losses and decoherence poses a serious problem for establishing entangled resources at distance or in a long computing network, since in a realistic transmission or computation the entanglement can be considerably degraded, thus preventing, for example, successful teleportation or dense coding. For this reason, the use of transformations which can increase the available entanglement by means of LOCC - although with some probability - is crucial for practical purposes. More generally, understanding entanglement transformations that are allowed by LOCC provides more insight in the structure and property of nonlocality, the most prominent character of quantum mechanics.

On entanglement transformations via LOCC there are two main results. The first is the seminal work by Nielsen 2] on deterministic transformations, which introduces majorization theory in this context. The second is the work by Vidal [3], which addresses the more general problem of probabilistic transformations. Such work is based on the approach of entanglement monotones, and gives conditions equivalent to weak majorization relations. The two approaches are completely disconnected, and for practical applications of the theory, a unified framework would be needed, especially in consideration that the more general treatment by Vidal is more abstract and less constructive than the Nielsen approach, which, however, is limited only to deterministic transformations. Furthermore, from the Vidal approach it is very difficult to recover the Nielsen treatment as a special case, and it is quite surprising that such approach ends up as a weak-majorization condition, without essentially using majorization theory. This motivates a derivation of the general nondeterministic LOCC transformations of pure states with an approach completely based on majoriza-

*URL: http://www.qubit.it tion theory, generalizing the Nielsen work [2].

In this paper, we present a general framework for entanglement transformations of bipartite pure states by means of LOCC. In Sec. II, we give a short and very simple proof of the theorem of Lo and Popescu [1], which is at the basis of the theory of all LOCC, and which states that given two separate parties, say Alice and Bob, all LOCC transformations on a pure bipartite state can be reduced to a contraction by Alice and a unitary transformation by Bob. We include our derivation of this theorem, since it is particularly simple and is based on a useful technique for operator transposition. In Sec. III, we derive the main theorem, which gives a necessary and sufficient condition for all entanglement transformations of pure states in terms of supermajorization conditions, generalizing the Nielsen approach, and recovering the result of Vidal. Here, we also provide a necessary condition for "pure contraction" transformations, namely those LOCC transformations in which the target state is achieved just for a single outcome of Alice measurement and a corresponding unitary on Bob side. Such a condition is written in terms of submajorization relation. In Sec. IV, explicit protocols to achieve pure, deterministic, and probabilistic transformations are given, using a method that gives the Alice contraction of the LOCC in terms of the Moore-Penrose pseudoinverse of the matrix of the entangled state. Section $\mathrm{V}$ concludes the paper summarizing the results.

\section{THE LO-POPESCU THEOREM}

For later convenience, we introduce here the main notation used in the paper. Given a linear operator $O$, we denote its Hermitian conjugate by $O^{\dagger}$. On a fixed basis, we write the complex conjugate and the transpose of $O$ as $O^{*}$ and $O^{\tau}$, respectively, so that $O^{\dagger}=\left(O^{*}\right)^{\tau}$. With the notation $O^{\ddagger}$ we denote the Moore-Penrose inverse of $O$. We recall that the Moore-Penrose inverse $O^{\ddagger}$ is the unique matrix that satisfies

$$
\begin{aligned}
& O O^{\ddagger} O=O, \quad O^{\ddagger} O O^{\ddagger}=O^{\ddagger}, \\
& O O^{\ddagger} \quad \text { and } \quad O^{\ddagger} O \quad \text { Hermitian . }
\end{aligned}
$$

From Eq. (1), it immediately follows that both $O O^{\ddagger}$ and $O^{\ddagger} O$ are orthogonal projectors, in particular, $O O^{\ddagger} \equiv P_{O}$ 
is the orthogonal projector over the range of $O \operatorname{Rng}(O)$, whereas $O^{\ddagger} O \equiv P_{O^{\dagger}}$ is the orthogonal projector over the support of $O \operatorname{Supp}(O) \doteq \operatorname{Ker}(O)^{\perp} \equiv \operatorname{Rng}\left(O^{\dagger}\right)$. We write the singular value decomposition (SVD) of $O$ as follows

$$
O=X_{O} \Sigma_{O} Y_{O}
$$

where $\Sigma_{O}$ denotes the diagonal matrix whose entries are the singular values $\sigma_{i}(O)$ of $O$ taken in a decreasing order, and $X_{O}, Y_{O}$ are unitary. The Moore-Penrose inverse $O^{\ddagger}$ then writes

$$
O^{\ddagger}=Y_{O}^{\dagger} \Sigma_{O}^{\ddagger} X_{O}^{\dagger},
$$

where $\Sigma_{O}^{\ddagger}$ is diagonal with entries $\sigma_{i}(O)^{-1}$ for $\sigma_{i}(O) \neq 0$, and zero entries for $\sigma_{i}(O)=0$.

We remember that a quantum measurement (with discrete spectrum) is generally described by a positive operator-valued measurement (POVM), namely by a resolution of the identity $\sum_{\lambda} M_{\lambda}^{\dagger} M_{\lambda}=1$, where each $\lambda$ corresponds to a possible outcome. Each operator $M_{\lambda}$ acts on the input states and is necessarily a contraction, namely it satisfies $\left\|M_{\lambda}\right\| \leq 1$, where $\|\ldots\|$ denotes the usual operator norm.

For bipartite pure states on the Hilbert space $\mathcal{H}_{1} \otimes \mathcal{H}_{2}$ we use the following notation $[8]$

$$
|A\rangle\rangle \equiv \sum_{i, j} a_{i j}|i\rangle_{1} \otimes|j\rangle_{2},
$$

where $\left\{|i\rangle_{1}\right\}$ and $\left\{|j\rangle_{2}\right\}$ are two chosen orthonormal bases for $\mathcal{H}_{1}$ and $\mathcal{H}_{2}$, respectively. Equation (4) introduces an isomorphism between vectors in $\mathcal{H}_{1} \otimes \mathcal{H}_{2}$ and $n \times m$ matrices, where $n$ and $m$ are the dimensions of $\mathcal{H}_{1}$ and $\mathcal{H}_{2}$. One can easily check the relation

$$
\left.A \otimes B|C\rangle\rangle=\left|A C B^{\tau}\right\rangle\right\rangle,
$$

where the transposition is defined with respect to the orthonormal basis $\left\{|j\rangle_{2}\right\}$. Finally, we use the notation $A \prec B$ for Hermitian operators $A$ and $B$ to denote the vector majorization relation [9] $\operatorname{eigv}(A) \prec \operatorname{eigv}(B)$, and in the same manner we will write $A \prec^{w} B$ and $A \prec_{w} B$ for super- and sub-majorization, respectively.

In the last part of this section, we provide a very short proof of the following theorem [1].

Theorem 1 All LOCC on a pure bipartite state $|\Psi\rangle$ can be reduced to a contraction by Alice and a unitary transformation by Bob. This resorts to the equivalence of any Bob contraction $M$ with the Alice contraction $N$ assisted by Bob unitary transformation $U$ as follows

$$
I \otimes M|\Psi\rangle\rangle=N \otimes U|\Psi\rangle\rangle,
$$

where

$$
N=K_{M \Psi^{\tau}} M K_{\Psi}, \quad U=K_{M \Psi^{\tau}}^{\dagger} K_{\Psi}^{\dagger},
$$

and $K_{O}$ is the unitary operator achieving the transposition of the operator $O$, namely,

$$
O^{\tau}=K_{O} O K_{O}^{*} .
$$

Proof. To prove that every LOCC can be reduced to an Alice contraction and a Bob unitary transformation, it is sufficient to prove the equivalence (6), since $a$ ) all possible elementary LOCC in a sequence will be reduced to an Alice contraction and a Bob unitary; $b$ ) the product of two contractions is a contraction; $c$ ) unitary transformations are particular cases of contraction.

Notice that given the SVD of any linear operator $O$ as in Eq. (2), one has

$$
\begin{aligned}
O^{\tau} & =Y_{O}^{\tau} \Sigma_{O} X_{O}^{\tau}=\left(Y_{O}^{\tau} X_{O}^{\dagger}\right) O\left(Y_{O}^{\tau} X_{O}^{\dagger}\right)^{*} \\
& \equiv K_{O} O K_{O}^{*},
\end{aligned}
$$

with $K_{O}=Y_{O}^{\tau} X_{O}^{\dagger}$. Using Eq. (5), Eq. (6) rewrites as follows

$$
\Psi M^{\tau}=N \Psi U^{\tau} .
$$

Then, from Eq. (9) one has

$$
\begin{aligned}
\Psi M^{\tau} & =\left(M \Psi^{\tau}\right)^{\tau}=K_{M \Psi^{\tau}}\left(M \Psi^{\tau}\right) K_{M \Psi^{\tau}}^{*} \\
& =K_{M \Psi^{\tau}} M K_{\Psi} \Psi K_{\Psi}^{*} K_{M \Psi^{\tau}}^{*},
\end{aligned}
$$

which is just Eq. (6) with $N$ and $U$ given as in Eq. (7).

\section{LOCC TRANSFORMATIONS FOR PURE STATES}

In this section, we will use the following useful lemmas.

Lemma 1 If $x \prec^{w} y$, then for some $v \quad x \prec v$ and $v \geq y$.

Proof. If $x \prec^{w} y$ one has for $2 \leq l \leq N$

$$
\sum_{i=l}^{N} x_{i} \geq \sum_{i=l}^{N} y_{i}
$$

and

$$
q \equiv \sum_{i=1}^{N} x_{i}>\sum_{i=1}^{N} y_{i} \equiv p
$$

Upon defining

$$
v=\left(q-p+y_{1}, y_{2}, \ldots, y_{N}\right)
$$

clearly one has $v \geq y$, and

$$
\begin{aligned}
\sum_{i=1}^{l} x_{i} & =q-\sum_{i=l+1}^{N} x_{i} \leq q-\sum_{i=l+1}^{N} y_{i} \\
& =q-p+\sum_{i=1}^{l} y_{i}=\sum_{i=1}^{l} v_{i}, \quad \forall l
\end{aligned}
$$

namely $x \prec v$. 
Lemma 2 If for some $u \quad x \geq u$ and $u \prec y$, then $x \prec^{w} y$.

Proof. One has

$$
\begin{aligned}
\sum_{i=l}^{N} x_{i} & \geq \sum_{i=l}^{N} u_{i}=\sum_{i=1}^{N} u_{i}-\sum_{i=1}^{l-1} u_{i} \\
& \geq \sum_{i=1}^{N} y_{i}-\sum_{i=1}^{l-1} y_{i}=\sum_{i=l}^{N} y_{i}, \quad \forall l
\end{aligned}
$$

namely, $x \prec^{w} y$.

We notice that both the above lemmas hold also in the reverse direction (for a proof see Ref. [10], pp. 11 and $123)$.

Moreover, we will make extensive use of the following theorem.

Theorem 2 (Uhlmann) For Hermitian operators $A$ and $B$, one has $A \prec B$ if and only if there are probabilities $q_{\lambda}$ and unitary operators $W_{\lambda}$ such that

$$
A=\sum_{\lambda} q_{\lambda} W_{\lambda}^{\dagger} B W_{\lambda}
$$

Proof. See Ref. [4], p. 575.

Theorem 2 relates majorization between Hermitian operators with a particular form of completely positive maps, namely those achievable through a random unitary evolution. In the terminology of quantum-information channels, such maps correspond to external-random-field channels that are a subclass of bistochastic channels (which send the identity operator into itself). For a qubit system $\left(\mathcal{H}=\mathcal{C}^{2}\right)$, the set of bistochastic and externalrandom-field channels coincide [11].

In the following, we derive the necessary and sufficient condition for all entanglement transformations of pure states in terms of supermajorization conditions. The theorem generalizes Nielsen approach $2 \mid$ and recovers the result of Vidal probabilistic transformations [3]. Moreover, we provide a necessary condition for "pure contraction" transformations, namely, those LOCC transformations in which the target state is achieved just for a single outcome of Alice measurement and a corresponding unitary on Bob side. In the proof a relevant role is played by the intermediate state (denoted in the following by $|Q\rangle\rangle$ ). In a transformation from $|A\rangle\rangle$ to $|B\rangle\rangle$, the state $|Q\rangle\rangle$ will be reached from $|A\rangle\rangle$ deterministically, whereas the final probabilistic transformation $|Q\rangle\rangle \rightarrow|B\rangle\rangle$ will be obtained through a pure contraction (for deterministic transformations one has $Q \equiv B$ ).

We are now ready to prove the following

Theorem 3 The state transformation $|A\rangle\rangle \rightarrow|B\rangle\rangle$ is possible by $L O C C$ iff

$$
A A^{\dagger} \prec{ }^{w} p B B^{\dagger},
$$

where $p \leq 1$ is the probability of achieving the transformation.
$A$ necessary condition to be satisfied is $\operatorname{rank}(A) \geq$ $\operatorname{rank}(B)$.

In particular, the transformation is deterministic $(p=1)$ iff $A A^{\dagger} \prec B B^{\dagger}$.

Finally, if there is a pure LOCC that achieves the state transformation with probability $p$, we must have

$$
p B B^{\dagger} \prec{ }_{w} A A^{\dagger} .
$$

Proof. Assume that $A A^{\dagger} \prec^{w} p B B^{\dagger}$. From Lemma 1 there is an operator $Q$ with $\Sigma_{Q}^{2} \geq p \Sigma_{B}^{2}$ and $A A^{\dagger} \prec Q Q^{\dagger}$. The state $|Q\rangle\rangle$ represents the intermediate state that can be achieved deterministically from $|A\rangle\rangle$. In fact, Uhlmann Theorem 2 guarantees the existence of a set of unitaries $W_{\lambda}$ and probabilities $q_{\lambda}$ such that

$$
A A^{\dagger}=\sum_{\lambda} q_{\lambda} W_{\lambda}^{\dagger} Q Q^{\dagger} W_{\lambda}
$$

Now, define the Alice measurement $\left\{M_{\lambda}\right\}$ such that

$$
M_{\lambda} \sqrt{A A^{\dagger}}=\sqrt{q_{\lambda} Q Q^{\dagger}} W_{\lambda},
$$

namely,

$$
M_{\lambda} A A^{\dagger} M_{\lambda}^{\dagger}=q_{\lambda} Q Q^{\dagger}, \quad \forall \lambda .
$$

We can always choose $M_{\lambda}$ such that $\operatorname{Supp}\left(M_{\lambda}\right) \subseteq \operatorname{Rng}(A)$, and show that $M_{\lambda}$ is a contraction, since

$$
\sqrt{A A^{\dagger}} \sum_{\lambda} M_{\lambda}^{\dagger} M_{\lambda} \sqrt{A A^{\dagger}}=A A^{\dagger}
$$

and so $\sum_{\lambda} M_{\lambda}^{\dagger} M_{\lambda}=P_{A} \leq I$. Then, there exists a set of unitary operators $U_{\lambda}$ such that $M_{\lambda} A U_{\lambda}^{\tau}=\sqrt{q_{\lambda}} Q$, namely,

$$
\left.\left.M_{\lambda} \otimes U_{\lambda}|A\rangle\right\rangle=\sqrt{q_{\lambda}}|Q\rangle\right\rangle,
$$

so that the transformation from $|A\rangle\rangle \rightarrow|Q\rangle\rangle$ can be achieved deterministically. Now, since $\Sigma_{Q}^{2} \geq p \Sigma_{B}^{2}$ one can define the contraction

$$
\tilde{N}=\sqrt{p} \sum_{l} \frac{\sigma_{l}(B)}{\sigma_{l}(Q)}|l\rangle\langle l|
$$

so that

$$
\tilde{N} \Sigma_{Q}^{2} \tilde{N}^{\dagger}=p \Sigma_{B}^{2} .
$$

By using the SVD of both $Q$ and $B$, Eq. (26) rewrites as follows

$$
\tilde{N} X_{Q}^{\dagger} Q Q^{\dagger} X_{Q} \tilde{N}^{\dagger}=p X_{B}^{\dagger} B B^{\dagger} X_{B},
$$

which means that there exists a unitary transformation $V$ such that

$$
N Q V^{\tau}=\sqrt{p} B,
$$


where $N=X_{B} \tilde{N} X_{Q}^{\dagger}$. Equation (28) is equivalent to the entanglement transformation

$$
N \otimes V|Q\rangle\rangle=\sqrt{p}|B\rangle\rangle
$$

namely, there is a pure LOCC occurring with probability $p$, which transforms the state $|Q\rangle\rangle$ into $|B\rangle\rangle$. As we have seen before, the transformation $|A\rangle\rangle \rightarrow|Q\rangle\rangle$ can be achieved deterministically, whence we conclude that $|A\rangle\rangle \rightarrow|B\rangle\rangle$ can be achieved with probability $p$, namely the statement of the first part of the theorem.

Reversely, assume that the transformation $|A\rangle\rangle \rightarrow|B\rangle\rangle$ can be achieved via LOCC with probability $p$. According to Theorem 1$]$ every LOCC is equivalent to a measurement performed by Alice followed by a conditional unitary by Bob. Therefore, the joint Alice-Bob state $R$ will evolve as follows:

$$
R \rightarrow \sum_{\lambda} M_{\lambda} \otimes U_{\lambda} R M_{\lambda}^{\dagger} \otimes U_{\lambda}^{\dagger},
$$

where

$$
\sum_{\lambda} M_{\lambda}^{\dagger} M_{\lambda}=I
$$

If the state $|A\rangle\rangle$ goes to $|B\rangle\rangle$, we must have for a subset S of the possible outcomes $\lambda$

$$
\left.\left.M_{\lambda} \otimes U_{\lambda}|A\rangle\right\rangle=\sqrt{p_{\lambda}}|B\rangle\right\rangle, \quad \forall \lambda \in \mathrm{S},
$$

where $\sum_{\lambda \in S} p_{\lambda}=p$ denotes the overall probability of the transformation $|A\rangle\rangle \rightarrow|B\rangle\rangle$. From Eq. (32), we need to have

$$
M_{\lambda} A U_{\lambda}^{\tau}=\sqrt{p_{\lambda}} B, \quad \forall \lambda \in \mathrm{S}
$$

and, therefore, each probability $p_{\lambda}$ is given by

$$
\left\|M_{\lambda} A U_{\lambda}^{\tau}\right\|_{2}^{2}=\left\|M_{\lambda} A\right\|_{2}^{2}=p_{\lambda},
$$

where $\|O\|_{2}=\sqrt{\operatorname{Tr}\left[O^{\dagger} O\right]}$ denotes the usual Frobenius norm. The condition (33) can be satisfied only if $\operatorname{rank}(A) \geq \operatorname{rank}(B)$, i.e. we can only decrease the Schmidt number of the entangled state.

From Eq. (32), one has $\forall \lambda \in \mathrm{S}$

$$
M_{\lambda} A A^{\dagger} M_{\lambda}^{\dagger}=p_{\lambda} B B^{\dagger},
$$

namely, by polar decomposition

$$
M_{\lambda} \sqrt{A A^{\dagger}}=\sqrt{M_{\lambda} A A^{\dagger} M_{\lambda}^{\dagger}} V_{\lambda}=\sqrt{p_{\lambda} B B^{\dagger}} V_{\lambda} .
$$

From Eq. (31), we have

$$
\begin{aligned}
\frac{1}{p} A A^{\dagger} & =\frac{1}{p} \sqrt{A A^{\dagger}} \sum_{\lambda} M_{\lambda}^{\dagger} M_{\lambda} \sqrt{A A^{\dagger}} \\
& \geq \frac{1}{p} \sqrt{A A^{\dagger}} \sum_{\lambda \in \mathrm{S}} M_{\lambda}^{\dagger} M_{\lambda} \sqrt{A A^{\dagger}},
\end{aligned}
$$

and using Eq. (36), we obtain

$$
\frac{1}{p} A A^{\dagger} \geq \sum_{\lambda \in \mathrm{S}} \frac{p_{\lambda}}{p} V_{\lambda}^{\dagger} B B^{\dagger} V_{\lambda} \equiv Q Q^{\dagger}
$$

By Uhlmann Theorem 2 we have

$$
Q Q^{\dagger} \prec B B^{\dagger}
$$

and from Lemma 2] we get the statement, namely, $A A^{\dagger} \prec{ }^{w} p B B^{\dagger}$.

We now prove the last part of the theorem, regarding the pure LOCC case. In a pure contraction transformation the target state is achieved just for a single outcome of Alice measurement and a unitary performed by Bob. Such a transformation that occurs with probability $p$ is given by

$$
M \otimes U|A\rangle\rangle=\sqrt{p}|B\rangle\rangle
$$

and we need to have

$$
\begin{aligned}
M A U^{\tau} & =\sqrt{p} B, \\
\left\|M A U^{\tau}\right\|_{2}^{2} & =\|M A\|_{2}^{2}=p .
\end{aligned}
$$

Again, this is possible if $\operatorname{rank}(A) \geq \operatorname{rank}(B)$. Using the SVD of $A$ and $B$ as in Eq. (2), Eq. (41) rewrites in terms of the diagonal matrices $\Sigma_{A}$ and $\Sigma_{B}$ as follows

$$
\tilde{M} \Sigma_{A} \tilde{U}=\sqrt{p} \Sigma_{B}
$$

with

$$
\tilde{M}=X_{B}^{\dagger} M X_{A}, \quad \tilde{U}=Y_{A} U^{\tau} Y_{B}^{\dagger}
$$

Equation (43) leads to

$$
\tilde{M} \Sigma_{A}^{2} \tilde{M}^{\dagger}=p \Sigma_{B}^{2}
$$

namely,

$$
\sum_{k} S_{k l} \sigma_{k}^{2}(A)=p \sigma_{l}^{2}(B)
$$

where $S_{k l} \doteq|\langle l|\tilde{M}| k\rangle|^{2}$ is a substochastic matrix [10], since

$$
\begin{aligned}
& \sum_{k} S_{k l}=\left\langle l\left|\tilde{M} \tilde{M}^{\dagger}\right| l\right\rangle \leq\left\|M^{\dagger}\right\|^{2} \leq 1, \\
& \sum_{l} S_{k l}=\left\langle k\left|\tilde{M}^{\dagger} \tilde{M}\right| k\right\rangle \leq\|M\|^{2} \leq 1 .
\end{aligned}
$$

Since Eq. (46) with $S$ substochastic is equivalent [10] to $p \sigma^{2}(B) \prec_{w} \sigma^{2}(A)$, namely $p B B^{\dagger} \prec_{w} A A^{\dagger}$, we have proved that Eq. (19) is a necessary condition for the LOCC transformation (40), namely, the last statement of the theorem. 


\section{EXPLICIT PROTOCOLS}

\section{A. Pure transformation}

Pure LOCC transformations are achieved by a single contraction on Alice side, assisted by a unitary by Bob. These are the most general one-way LOCC operations. In this case, we have the following theorem.

Theorem 4 The transformation $|A\rangle\rangle \rightarrow|B\rangle\rangle$ can be achieved with probability $p$ by a pure LOCC transformation iff one can find a unitary operator $U$ and linear operator $N$ such that

$$
M=\sqrt{p} B U^{*} A^{\ddagger}+N\left(I-A A^{\ddagger}\right)
$$

is a contraction. The transformation is then obtained as

$$
M \otimes U|A\rangle\rangle=\sqrt{p}|B\rangle\rangle
$$

Proof. Notice that Eq. (50) is equivalent to the identity

$$
M A=\sqrt{p} B U^{*}
$$

Since both sides of the identity must have the same kernel it follows that

$$
M A=\sqrt{p} B U^{*} A^{\ddagger} A,
$$

and, multiplying both sides by $A^{\ddagger}$, we have

$$
M P_{A}=\sqrt{p} B U^{*} A^{\ddagger} A A^{\ddagger}=\sqrt{p} B U^{*} A^{\ddagger} P_{A}=\sqrt{p} B U^{*} A^{\ddagger} .
$$

The general solution of the last equation is

$$
M=\sqrt{p} B U^{*} A^{\ddagger} P_{A}+N\left(I-P_{A}\right)
$$

with arbitrary $N$ and, in fact, one can easily check that

$$
M A=\sqrt{p} B U^{*} A^{\ddagger} A=\sqrt{p} B U^{*} .
$$

The unitary $U$ and the operator $N$ should be taken such that $M$ is a contraction. This is not always possible. However, a sufficient condition is

$$
p \Sigma_{B}^{2} \leq \Sigma_{A}^{2} \text {. }
$$

In fact, by taking $N=0$ and $U=Y_{B}^{T} Y_{A}^{*}$, one has $M=\sqrt{p} X_{B} \Sigma_{B} \Sigma_{A}^{\ddagger} X_{A}^{\dagger}$, and then, for Eq. (156), $\|M\|=$ $\sqrt{p}\left\|\Sigma_{B} \Sigma_{A}^{\ddagger}\right\| \leq 1$.

\section{B. Deterministic transformation}

In the entanglement transformations, the first part of the protocol is a deterministic transformation from $|A\rangle\rangle$ to $|Q\rangle\rangle$. The majorization relation $A A^{\dagger} \prec Q Q^{\dagger}$ implies
Theorem[2] namely the existence of a set of unitaries $W_{\lambda}$ and probabilities $q_{\lambda}$ such that

$$
A A^{\dagger}=\sum_{\lambda} q_{\lambda} W_{\lambda}^{\dagger} Q Q^{\dagger} W_{\lambda} .
$$

In order to construct explicitly the protocol, one needs to find contractions $M_{\lambda}$ and unitaries $U_{\lambda}$ versus the unitary operators $W_{\lambda}$ that appear in Eq. (57) such that one has

$$
\left.\left.M_{\lambda} \otimes U_{\lambda}|A\rangle\right\rangle=\sqrt{q_{\lambda}}|Q\rangle\right\rangle
$$

We have seen that the general form of the solution of Eq. (58) is given by

$$
M_{\lambda}=\sqrt{q_{\lambda}} Q U_{\lambda}^{*} A^{\ddagger}+N_{\lambda}\left(1-A A^{\ddagger}\right) .
$$

We have now the following theorem

Theorem 5 In Eq. (59), we can always take

$$
N_{\lambda}=0, \quad U_{\lambda}^{*}=Y_{Q}^{\dagger} X_{Q}^{\dagger} W_{\lambda} X_{A} Y_{A}
$$

where $X_{O}, Y_{O}$ are the operators defined in Eq. (2) such that $M_{\lambda}$ is a contraction and Eq. (58) is satisfied.

Proof. Substituting Eq. (60) in Eq. (59), one has

$$
\begin{aligned}
& \sum_{\lambda} M_{\lambda}^{\dagger} M_{\lambda}=\sum_{\lambda} q_{\lambda}\left(A^{\ddagger}\right)^{\dagger} U_{\lambda}^{\tau} Q^{\dagger} Q U_{\lambda}^{*} A^{\ddagger} \\
& =\sum_{\lambda} q_{\lambda}\left(A^{\ddagger}\right)^{\dagger} Y_{A}^{\dagger} X_{A}^{\dagger} W_{\lambda}^{\dagger} X_{Q} Y_{Q} Q^{\dagger} Q Y_{Q}^{\dagger} X_{Q}^{\dagger} W_{\lambda} X_{A} Y_{A} A^{\ddagger} \\
& =\sum_{\lambda} q_{\lambda}\left(A^{\ddagger}\right)^{\dagger} Y_{A}^{\dagger} X_{A}^{\dagger} W_{\lambda}^{\dagger} Q Q^{\dagger} W_{\lambda} X_{A} Y_{A} A^{\ddagger},
\end{aligned}
$$

and using Eq. (57), one has

$$
\begin{aligned}
& \sum_{\lambda} M_{\lambda}^{\dagger} M_{\lambda}=\left(A^{\ddagger}\right)^{\dagger} Y_{A}^{\dagger} X_{A}^{\dagger} A A^{\dagger} X_{A} Y_{A} A^{\ddagger} \\
& =\left(A^{\ddagger}\right)^{\dagger} A^{\dagger} A A^{\ddagger}=\left(A A^{\ddagger}\right)^{\dagger} A A^{\ddagger}=A A^{\ddagger}=P_{A} \leq I .
\end{aligned}
$$

Hence, $M_{\lambda}$ are contractions. The completeness of the measurement can be guaranteed by the further contraction

$$
M_{0}=V\left(I-A A^{\ddagger}\right),
$$

where $V$ is an arbitrary unitary operator.

For any outcome $\lambda$ on Alice side, Bob performs the unitary $U_{\lambda}$. Using Eqs. (59) and (60), one has

$$
\begin{aligned}
M_{\lambda} A U_{\lambda}^{\tau} & =\sqrt{q_{\lambda}} Q Y_{Q}^{\dagger} X_{Q}^{\dagger} W_{\lambda} X_{A} Y_{A} A^{\ddagger} A Y_{A}^{\dagger} X_{A}^{\dagger} W_{\lambda}^{\dagger} X_{Q} Y_{Q} \\
& =\sqrt{q_{\lambda}} Q Y_{Q}^{\dagger} X_{Q}^{\dagger} W_{\lambda} A A^{\ddagger} W_{\lambda}^{\dagger} X_{Q} Y_{Q} .
\end{aligned}
$$

From Eq. (57), it follows that $\operatorname{Rng}\left(W_{\lambda}^{\dagger} Q Q^{\dagger} W_{\lambda}\right) \subseteq$ $\operatorname{Rng}\left(A A^{\dagger}\right) \equiv \operatorname{Rng}(A)$, namely,

$$
P_{A}=P_{A A^{\dagger}} \geq W_{\lambda}^{\dagger} P_{Q Q^{\dagger}} W_{\lambda}, \quad \forall \lambda .
$$


Hence, by multiplying both sides of Eq. (65) on the left by $Y_{Q}^{\dagger} X_{Q}^{\dagger} W_{\lambda}$ and on the right by $W_{\lambda}^{\dagger} X_{Q} Y_{Q}$, one obtains

$$
\begin{aligned}
Y_{Q}^{\dagger} X_{Q}^{\dagger} W_{\lambda} P_{A} W_{\lambda}^{\dagger} X_{Q} Y_{Q} & \geq Y_{Q}^{\dagger} X_{Q}^{\dagger} P_{Q Q^{\dagger}} X_{Q} Y_{Q} \\
& =P_{Q^{\dagger} Q}=P_{Q^{\dagger}}
\end{aligned}
$$

The projector on $\operatorname{Rng}\left(Q^{\dagger}\right)$ coincides with the projector on $\operatorname{Ker}(Q)^{\perp} \equiv \operatorname{Supp}(Q)$. So Eq. (64) gives

$$
M_{\lambda} A U_{\lambda}^{\tau}=\sqrt{q_{\lambda}} Q
$$

which is equivalent to Eq. (58).

According to our derivation, given explicitly Eq. (57), one can perform the contractions $M_{\lambda}$ and the unitaries $U_{\lambda}$ to achieve the transformation $\left.\left.|A\rangle\right\rangle \rightarrow|Q\rangle\right\rangle$. The problem of looking for a POVM with minimum number of outcomes (thus, minimizing the amount of classical information sent to Bob) is reduced to find the transformation (57) with minimum number of unitaries.

One can resort to a constructive algorithm to find a bistochastic matrix $D$ that relates the vectors $\sigma^{2}(A)$ and $\sigma^{2}(Q)$ of the singular values of $A$ and $Q$, namely

$$
\vec{\sigma}^{2}(A)=D \vec{\sigma}^{2}(Q) .
$$

Then Birkhoff theorem 10] allows to write $D$ as a convex combination of permutation matrices

$$
D=\sum_{\lambda} q_{\lambda} \Pi_{\lambda}
$$

In terms of $\Sigma_{A}$ and $\Sigma_{Q}$ one has

$$
\Sigma_{A}^{2}=\sum_{\lambda} q_{\lambda} \Pi_{\lambda}^{\dagger} \Sigma_{Q}^{2} \Pi_{\lambda},
$$

where $\Pi_{\lambda}=\sum_{l}|l\rangle\left\langle\Pi_{\lambda}(l)\right|$. In this way one obtains Eq. (57), with $W_{\lambda}=X_{Q} \Pi_{\lambda} X_{A}^{\dagger}$. Using the corresponding expressions for the contractions $M_{\lambda}$ and unitaries $U_{\lambda}$ one recovers the result of Ref. [12]. Notice that Caratheodory's theorem always allows to reduce the number of permutations in Eq. (70) to $(d-1)^{2}+1$, for $d$-dimensional Alice Hilbert space.

\section{Probabilistic transformation}

The second part of the protocol, namely, the contraction that provides the state $|B\rangle\rangle$ from $|Q\rangle\rangle$, is needed only for probabilistic transformations. It is a pure contraction given by

$$
\left.\left.N \otimes V|Q\rangle\rangle=\left|N Q V^{\tau}\right\rangle\right\rangle=\sqrt{p}|B\rangle\right\rangle
$$

with

$$
N=\sqrt{p} X_{B} \Sigma_{B} \Sigma_{Q}^{\ddagger} X_{Q}^{\dagger}
$$

and

$$
V^{\tau}=Y_{Q}^{\dagger} Y_{B}
$$

In fact for Lemma 1 one has $\Sigma_{Q}^{2} \geq p \Sigma_{B}^{2}$, which implies that $\Sigma_{B} \Sigma_{Q}^{\ddagger} \Sigma_{Q}=\Sigma_{B}$. Then

$$
\begin{aligned}
N Q V^{\tau} & =\sqrt{p} X_{B} \Sigma_{B} \Sigma_{Q}^{\ddagger} \Sigma_{Q} Y_{B} \\
& =\sqrt{p} X_{B} \Sigma_{B} Y_{B}=\sqrt{p} B .
\end{aligned}
$$

\section{CONCLUSIONS}

In this paper, we presented a general theoretical framework for both deterministic and probabilistic entanglement transformations of bipartite pure states achieved via LOCC transformations. We have generalized Nielsen work based on majorization theory [2] in order to include the more general results by Vidal [3], which were based on the approach of the entanglement monotones. The main theorem gives an if and only if condition for all entanglement transformations of pure states in terms of super-majorization conditions. We also provided a necessary submajorization condition for pure transformations, which allows to write each contraction in terms of the Moore-Penrose pseudoinverse of the matrix of the entangled state. This led to explicit protocols to achieve pure, deterministic, and probabilistic LOCC.

We notice that all theorems have been derived in finite dimensions, but they can be easily extended to infinite dimensions for contractions that are compact operators and for normalized entangled states corresponding to Hilbert-Schmidt operators. Thus, our results also apply to continuous variables.

\section{Acknowledgments}

We are grateful to Shashank Virmani for useful discussions. This work has been jointly founded by the EC under the program EQUIP (Contract No. IST-1999-11053) and ATESIT (Contract No. IST-2000-29681). G. M. D. acknowledges support from the Department of Defense Multidisciplinary University Research Initiative (MURI) program administered by the Army Research Office under Grant No. DAAD19-00-1-0177.
[1] H.-K. Lo and S. Popescu, Phys. Rev. A 63, 022301 (2001).
[2] M. A. Nielsen, Phys. Rev. Lett. 83, 436 (1999). 
[3] G. Vidal, Phys. Rev. Lett. 83, 1046 (1999).

[4] I. L. Chuang and M. A. Nielsen, Quantum Information and Quantum Computation, Cambridge University Press, Cambridge UK, 2000.

[5] Introduction to Quantum Computation and Information, edited by H-K Lo, S. Popescu, and T. Spiller (World Scientific, Singapore, 1998).

[6] C. H. Bennett, G. Brassard, C. Crepeau, R. Jozsa, A. Peres, and W. K. Wootters, Phys. Rev. Lett. 70, 1895 (1993).

[7] A. K. Ekert, Phys. Rev. Lett. 67, 661 (1991).

[8] G. M. D'Ariano, P. Lo Presti, and M. F. Sacchi, Phys. Lett. A 272, 32 (2000).

[9] We say that the vector $y$ majorizes the vector $x$ and write $x \prec y$ iff, upon arranging their elements in decreasing order $\left(x_{1} \geq x_{2} \geq \ldots \geq x_{N}\right.$ and $\left.y_{1} \geq y_{2} \geq \ldots \geq y_{N}\right)$, one has

$$
\sum_{i=1}^{l} x_{i} \leq \sum_{i=1}^{l} y_{i} \forall l \in[1, N-1] \text { and } \sum_{i=1}^{N} x_{i}=\sum_{i=1}^{N} y_{i} .
$$

Submajorization and supermajorization are denoted by $x \prec w \quad y$ and $x \prec^{w} y$, and are defined by the weaker conditions:

$$
\begin{aligned}
& x \prec_{w} y \Longleftrightarrow \\
& \sum_{i=1}^{l} x_{i} \leq \sum_{i=1}^{l} y_{i} \forall l \in[1, N-1] \text { and } \sum_{i=1}^{N} x_{i}<\sum_{i=1}^{N} y_{i} ; \\
& x \prec^{w} y \Longleftrightarrow \\
& \sum_{i=l}^{N} x_{i} \geq \sum_{i=l}^{N} y_{i} \forall l \in[2, N] \text { and } \sum_{i=1}^{N} x_{i}>\sum_{i=1}^{N} y_{i} .
\end{aligned}
$$

See, for example, R. Bhatia, Matrix Analysis, Springer Graduate Texts in Mathematics Vol. 169 (Springer, New York, 1996).

[10] A. W. Marshall and I. Olkin, Inequalities: Theory of Majorization and Its Applications, Academic Press, New York, 1979.

[11] P. Badziąg, M. Horodecki, P. Horodecki, and R. Horodecki, Phys. Rev. A 62, 012311 (2000).

[12] J. G. Jensen and R. Schack, Phys. Rev. A 63, 062303 (2001). 